Gaugi ng away the el ectron el ectron i nt er act i on by the I ocal phase of compl ex wave functions in two- el ectron systems

\begin{tabular}{|l|l|}
\hline 著者 & OHTA Kat suhi sa \\
\hline $\begin{array}{l}\text { j our nal or } \\
\text { publ i cat } \mathrm{i} \text { on } \mathrm{t} \text { i t l e }\end{array}$ & J our nal of Nat hemat i cal Chemi st ry \\
\hline vol ume & 55 \\
\hline number & 1 \\
\hline page $\mathrm{r}$ ange & $98-104$ \\
\hline year & $2017-01$ \\
\hline URL & ht t p: //hdl . handl e. net /10258/00009462 \\
\hline
\end{tabular}




\title{
Gauging away the electron-electron interaction by the local phase of complex wave functions in two-electron systems
}

\author{
Katsuhisa Ohta $^{1}$ \\ 1 Applied Chemistry Research Unit, Graduate School of Engineering, \\ Muroran Institute of Technology, Muroran, Hokkaido, 050-8585, Japan \\ E-mail: ohta@mmm.muroran-it.ac.jp
}

\begin{abstract}
The electron-electron interaction is eliminated in the expectation values of the electronic Hamiltonian for two-electron systems. The part of the Hamiltonian referring to the repulsive interaction is gauged away by the local phase of the complex wave functions, much like a gauge field transformation, thereby leading to a one-electron Hamiltonian. Despite the appearance of complex wave functions, the expectation values of the total momentum operator vanish and Löwdin's criterion holds for the stationary states.
\end{abstract}

PACS numbers: 


\section{COMPLEX WAVE FUNCTIONS FOR STATIONARY STATES}

The appearance of complex wave functions is one of the mathematical necessities required in solving the time-dependent Schrödinger equation[1]. Moreover, for non-degenerate stationary states, the complex degree of freedom of wave functions has sometimes been introduced for computational convenience. For example, complex one-electron orbitals in a single-determinantal wave function are convenient in describing energy crossings [2, 3].

The appropriateness of real functions for many-electron wave functions corresponding to stationary states has been discussed based on physical and mathematical requirements. First, the use of real wave functions is inevitable if Löwdin's criterion for stationary states is hold; that is, by a result of Ehrenfest's theorem, the conservation of total momentum is

$$
\langle\Psi|\hat{P}| \Psi\rangle=0
$$

Hence the use of the real wave functions is by no means solely for mathematical convenience[4]. Second, if we write $\Psi=\operatorname{Re} \Psi+i \operatorname{Im} \Psi$ and set $\bar{H}_{[\Psi]}=\frac{\langle\Psi|\hat{H}| \Psi\rangle}{\langle\Psi \mid \Psi\rangle}$, we have

$$
\operatorname{Min}\left(\bar{H}_{[R e \Psi]}, \bar{H}_{[\operatorname{Im} \Psi]}\right) \leq \bar{H}_{[\Psi]},
$$

obtained from variational calculations for the stationary states. Despite the substantial complex freedom, the wave function does not provide a minimum energy $[5,6]$.

In this paper we report another feature of complex wave functions for stationary states. The electron-electron interaction is formally eliminated from the expectation values of the electronic Hamiltonian for two-electron systems. In particle physics[7, 8], interactions between particles are introduced by local-gauge transformations. Conversely, the interactions can be gauged away formally by wave functions with local phase. We have adopted similar equations to eliminate the electron-electron interaction in the expectation values. Despite complex wave functions, the expectation values of the total momentum operator vanish and Löwdin's criterion Eq. (1) for stationary states is satisfied. The difficulty in applications to systems with more than three electrons is also discussed. 


\section{GAUGING AWAY THE POSITIVE POTENTIALS BY THE LOCAL PHASE OF COMPLEX WAVE FUNCTIONS}

\section{A. Local phase of complex wave functions}

We consider complex wave functions corresponding to stationary states of one-particle systems. A polar decomposition of $\Psi(\boldsymbol{r})$ is introduced with local phase $S(\boldsymbol{r})$,

$$
\Psi(\boldsymbol{r})=e^{i S(\boldsymbol{r})} \Phi(\boldsymbol{r})
$$

where $S$ and $\Phi$ are real functions. The wave function $\Phi$ is also normalized with the standard boundary condition for bounded systems

$$
\lim _{|\boldsymbol{r}| \rightarrow \infty} \Phi(\boldsymbol{r})=0
$$

The Schrödinger equation with the vector potential $\boldsymbol{A}$ is

$$
\left[\frac{1}{2}(\hat{p}-\boldsymbol{A})^{2}+V-E\right] \Psi(\boldsymbol{r})=0 .
$$

If $V=0$ and $\boldsymbol{A}=0$ are set, we have

$$
\left[-\frac{1}{2} \Delta-E\right] \Psi(\boldsymbol{r})=0
$$

and

$$
\left[-\frac{1}{2} \Delta+\frac{1}{2} \nabla S \cdot \nabla S-\frac{i}{2}(\Delta S+2 \nabla S \nabla)-E\right] \Phi(\boldsymbol{r})=0 .
$$

If we multiply Eq. (7) by $\Phi$ from the left and integrate, the contribution from the imaginary part of Eq. (7) is rewritten as

$$
\int_{V} \Phi(\Delta S+2 \nabla S \nabla) \Phi d v=\int_{V} \nabla\left(\Phi^{2} \nabla S\right) d v=\oint_{\Sigma} \Phi^{2} \nabla S d \sigma,
$$

according to the Gauss theorem. If $\Phi^{2} \nabla S$ vanishes in infinity with the boundary condition Eq. (4), the surface integral is equal to zero. So we have

$$
\left\langle\Psi\left|-\frac{1}{2} \Delta\right| \Psi\right\rangle=\left\langle\Phi\left|-\frac{1}{2} \Delta+\frac{1}{2} \nabla S \cdot \nabla S\right| \Phi\right\rangle,
$$

in the form of expectation values. In the right-hand side of Eq. (9), the second term can be seen as a new positive potential for $\Phi$, which is originated from the localized phase $S$ as

$$
\hat{V}_{+}=\frac{1}{2} \nabla S \cdot \nabla S
$$


Eq. (10) is considered to be another proof of the inequality Eq. (2).

Conversely, we can absorb some positive part of the potential into the local phase $S$ of the complex wave function. If a positive potential $\hat{V}_{+}$is separated in a given Hamiltonian $\hat{H}$ as

$$
\hat{H}=\hat{H}_{0}+\hat{V}_{+}
$$

we can then eliminate $\hat{V}_{+}$in the expectation value of the Hamiltonian $\hat{H}$ with an appropriately prepared local phase $S$ by Eq. (10). Note that the diagonal element of the density matrix with the complex wave function $\Psi$ remains equal to that with the real function $\Phi$ as

$$
\begin{aligned}
\left.\rho_{\Psi}\left(\boldsymbol{r}, \boldsymbol{r}^{\prime}\right)\right|_{\boldsymbol{r}=\boldsymbol{r}^{\prime}} & =\left.\Psi^{*}(\boldsymbol{r}) \Psi\left(\boldsymbol{r}^{\prime}\right)\right|_{\boldsymbol{r}=\boldsymbol{r}^{\prime}} \\
& =\left.e^{-i S(\boldsymbol{r})} \Phi(\boldsymbol{r}) e^{i S\left(\boldsymbol{r}^{\prime}\right)} \Phi\left(\boldsymbol{r}^{\prime}\right)\right|_{\boldsymbol{r}=\boldsymbol{r}^{\prime}}=\left.\rho_{\Phi}\left(\boldsymbol{r}, \boldsymbol{r}^{\prime}\right)\right|_{\boldsymbol{r}=\boldsymbol{r}^{\prime}}
\end{aligned}
$$

\section{B. One-dimensional systems}

Here we exemplify the use of Eq. (10) with a simple system. If we consider a power law in the coordinate $x$ for the local phase,

$$
S(x)=\alpha|x|^{\beta}
$$

we can generate the positive potential

$$
\hat{V}_{+}(x ; \alpha, \beta)=\frac{1}{2} \nabla S(x) \cdot \nabla S(x)=\frac{1}{2} \alpha^{2} \beta^{2}|x|^{2(\beta-1)} .
$$

We notice that the positive potential Eq. (14) has even parity under space inversion symmetry. Moreover, if the system is subjected only to a positive potential $\hat{V}_{+}(x ; \alpha, \beta)$ with the asymptotic behavior $\lim _{x \rightarrow \pm \infty} \hat{V}_{+}(x ; \alpha, \beta)=0$, the entire system becomes unbounded. In this instance, it is impossible to gauge away the positive potential by the local phase of the complex wave function using the boundary condition Eq. (4). Therefore, $\hat{V}_{+}(x ; \alpha, \beta)$ should satisfy the asymptotic behavior as $\lim _{x \rightarrow \pm \infty} \hat{V}_{+}(x ; \alpha, \beta)=\infty$. This condition is ensured if $\beta>1$ in Eq. (14).

For example, if we consider a harmonic oscillator which corresponds to $\beta=2$, the Hamiltonian is

$$
\hat{H}=-\frac{1}{2} \frac{d^{2}}{d x^{2}}+\frac{k}{2} x^{2}=\hat{H}_{0}+\frac{k}{2} x^{2}, \quad(k>0) .
$$


The local phase which absorbs this harmonic potential is

$$
S(x)= \pm \frac{\sqrt{k}}{2} x^{2}
$$

and the complex wave function Eq. (3) is written as

$$
\Psi(x)=e^{i S(x)} \Phi(x)=e^{ \pm i \frac{\sqrt{k}}{2} x^{2}} \Phi(x) .
$$

The energy expectation value formally reduces to that of the free-particle Hamiltonian $\hat{H}_{0}$ with complex wave function $\Psi$; that is

$$
\begin{aligned}
\mathcal{E} & =\langle\Phi|\hat{H}| \Phi\rangle=\left\langle\Phi\left|-\frac{1}{2} \frac{d^{2}}{d x^{2}}+\frac{k}{2} x^{2}\right| \Phi\right\rangle=\left\langle\Psi\left|-\frac{1}{2} \frac{d^{2}}{d x^{2}}\right| \Psi\right\rangle, \\
& =\left\langle\Psi\left|\hat{H}_{0}\right| \Psi\right\rangle .
\end{aligned}
$$

If we use exact wave functions of the harmonic oscillator for $\Phi$, we have

$$
\Psi_{n}(x)=e^{i S(x)} \Phi_{n}(x)=\sqrt{\frac{1}{2^{n} n !}}\left(\frac{\sqrt{k}}{\pi}\right)^{\frac{1}{4}} H_{n}\left(k^{\frac{1}{4}} x\right) e^{-(1 \pm i) \frac{\sqrt{k}}{2} x^{2}}
$$

where $H_{n}$ are Hermite polynomials. Although the wave function is complex, the expectation value of the momentum is zero,

$$
\begin{aligned}
\bar{p}_{x} & =\int_{-\infty}^{\infty} \Psi^{*}(x)\left(-i \frac{d}{d x}\right) \Psi(x) d x \\
& =\int_{-\infty}^{\infty} \Phi^{2}\left(\frac{d S}{d x}\right) d x-i \int_{-\infty}^{\infty} \Phi\left(\frac{d \Phi}{d x}\right) d x=0,
\end{aligned}
$$

where the first term $\int_{-\infty}^{\infty} \Phi^{2}\left(\frac{d S}{d x}\right) d x$ vanishes, because the parity of $\Phi^{2}$ is even under spatial inversion symmetry of the potential Eq. (14) and the parity of $\left(\frac{d S}{d x}\right)$ is odd by Eq. (16). After partial integration, the second term $\int_{-\infty}^{\infty} \Phi\left(\frac{d \Phi}{d x}\right) d x$ also vanishes on applying by the boundary condition Eq. (4). Löwdin's criterion Eq. (1) for stationary states is satisfied.

C. Elimination of the repulsive electron-electron interaction in two-electron systems

We next consider a two-electron system for which the electronic Hamiltonian is written as

$$
\begin{aligned}
\hat{H} & =\sum_{i=1}^{2}\left(-\frac{1}{2} \boldsymbol{\nabla}_{i} \cdot \boldsymbol{\nabla}_{i}-\sum_{A=1}^{M} \frac{Z_{A}}{\left|\boldsymbol{r}_{i}-\boldsymbol{R}_{A}\right|}\right)+\frac{1}{\left|\boldsymbol{r}_{1}-\boldsymbol{r}_{2}\right|} \\
& =\hat{H}_{0}+\frac{1}{\left|\boldsymbol{r}_{1}-\boldsymbol{r}_{2}\right|}
\end{aligned}
$$


in au, where $\hat{H}_{0}$ is just a sum of one-electron operators. If only a repulsive potential $\hat{V}_{+}=$ $\frac{1}{\left|\boldsymbol{r}_{1}-\boldsymbol{r}_{2}\right|}$ is present, the Hamiltonian does not correspond to a bounded system and we cannot use boundary conditions such as Eq. (4). However, there are attractive nuclear-potentials in the one-electron Hamiltonian $\hat{H}_{0}$ for which the whole system is a bounded system. We then assume an asymptotic behavior for the two-electron wave function $\Phi\left(\boldsymbol{r}_{1}, \boldsymbol{r}_{2}, \sigma_{1}, \sigma_{2}\right)=$ $\Phi\left(x_{1}, y_{1}, z_{1}, x_{2}, y_{2}, z_{2}, \sigma_{1}, \sigma_{2}\right)$,

$$
\lim _{x_{1} \rightarrow \pm \infty} \Phi\left(x_{1}, y_{1}, z_{1}, x_{2}, y_{2}, z_{2}, \sigma_{1}, \sigma_{2}\right)=0
$$

and similarly for the other coordinates; here $\sigma_{1}$ and $\sigma_{2}$ denote electronic spin coordinates.

The local phase $S\left(\boldsymbol{r}_{1}, \boldsymbol{r}_{2}\right)$ is presumed symmetric under the exchange of electrons,

$$
S\left(\boldsymbol{r}_{1}, \boldsymbol{r}_{2}\right)=\alpha\left[\left(x_{1}-x_{2}\right)^{2}+\left(y_{1}-y_{2}\right)^{2}+\left(z_{1}-z_{2}\right)^{2}\right]^{\frac{1}{4}}=\alpha\left|\boldsymbol{r}_{1}-\boldsymbol{r}_{2}\right|^{\frac{1}{2}}
$$

by imposing the Pauli principle on $\Phi\left(\boldsymbol{r}_{1}, \boldsymbol{r}_{2}, \sigma_{1}, \sigma_{2}\right)$. The positive potential for the local phase Eq. (23) becomes

$$
\begin{aligned}
\frac{1}{2} \boldsymbol{\nabla} S \cdot \boldsymbol{\nabla} S & =\frac{1}{2} \sum_{i=1}^{2}\left[\left(\frac{\partial S}{\partial x_{i}}\right)^{2}+\left(\frac{\partial S}{\partial y_{i}}\right)^{2}+\left(\frac{\partial S}{\partial z_{i}}\right)^{2}\right] \\
& =\left(\frac{\alpha^{2}}{4}\right) \frac{1}{\left|\boldsymbol{r}_{1}-\boldsymbol{r}_{2}\right|} .
\end{aligned}
$$

Then, setting $\alpha= \pm 2$, the repulsive interaction is absorbed into the local phase of the complex wave function as in gauge field transformations, and leads to a one-electron Hamiltonian $\hat{H}_{0}$ operator $[7,8]$. The complex electronic wave function becomes

$$
\begin{aligned}
\Psi\left(\boldsymbol{r}_{1}, \boldsymbol{r}_{2}, \sigma_{1}, \sigma_{2}\right) & =e^{i S\left(\boldsymbol{r}_{1}, \boldsymbol{r}_{2}\right)} \Phi\left(\boldsymbol{r}_{1}, \boldsymbol{r}_{2}, \sigma_{1}, \sigma_{2}\right) \\
& =e^{ \pm i 2 \sqrt{\left|\boldsymbol{r}_{1}-\boldsymbol{r}_{2}\right|}} \Phi\left(\boldsymbol{r}_{1}, \boldsymbol{r}_{2}, \sigma_{1}, \sigma_{2}\right)
\end{aligned}
$$

The expectation value for the Hamiltonian $\hat{H}$ is formally reduced to that for the one-electron Hamiltonian $\hat{H}_{0}$ with $\Psi$,

$$
\mathcal{E}=\langle\Phi|\hat{H}| \Phi\rangle=\left\langle\Phi\left|e^{-i S} \hat{H}_{0} e^{i S}\right| \Phi\right\rangle=\left\langle\Psi\left|\hat{H}_{0}\right| \Psi\right\rangle
$$

Eq. (25) is a local unitary of the wave function $\Phi$ to $\Psi$ that is part of a local gauge transformation. The transformation, however, has a definite form determined by the given Hamiltonian $\hat{H}$. Therefore, the freedom associated with the variational calculations remains only in $\Phi$. 
Next we consider the expectation value of the total momentum operator

$$
\hat{\boldsymbol{P}}=\hat{\boldsymbol{p}}_{1}+\hat{\boldsymbol{p}}_{2}=\left(-i \partial_{x_{1}}-i \partial_{x_{2}}, \quad-i \partial_{y_{1}}-i \partial_{y_{2}}, \quad-i \partial_{z_{1}}-i \partial_{z_{2}}\right)
$$

For brevity, we present the calculation for the $x$ component. Denoting other variables as $\tau=\left(y_{1}, z_{1}, y_{2}, z_{2}, \sigma_{1}, \sigma_{2}\right)$, we have

$$
\begin{aligned}
\bar{p}_{x_{1}} & =\int\left[\int_{-\infty}^{\infty} \int_{-\infty}^{\infty} \Psi^{*}\left(x_{1}, x_{2}, \tau\right)\left(-i \partial_{x_{1}}\right) \Psi\left(x_{1}, x_{2}, \tau\right) d x_{1} d x_{2}\right] d \tau \\
& =\int\left[\int_{-\infty}^{\infty} \int_{-\infty}^{\infty}\left(\partial_{x_{1}} S\right) \Phi^{2} d x_{1} d x_{2}\right] d \tau
\end{aligned}
$$

using the boundary condition Eq. (22). For the local phase Eq. (23), we have

$$
\begin{aligned}
\frac{\partial S}{\partial x_{1}} & =\left(\frac{\alpha}{2}\right)\left[\left(x_{1}-x_{2}\right)^{2}+\left(y_{1}-y_{2}\right)^{2}+\left(z_{1}-z_{2}\right)^{2}\right]^{-\frac{3}{4}}\left(x_{1}-x_{2}\right), \\
& =-\frac{\partial S}{\partial x_{2}}
\end{aligned}
$$

Hence the $x$ component of the total momentum vanishes as

$$
\bar{P}_{x}=\bar{p}_{x_{1}}+\bar{p}_{x_{2}}=\int\left[\int_{-\infty}^{\infty} \int_{-\infty}^{\infty}\left(\frac{\partial S}{\partial x_{1}}+\frac{\partial S}{\partial x_{2}}\right) \Phi^{2} d x_{1} d x_{2}\right] d \tau=0 .
$$

The other components also vanish in the same way and we have collectively

$$
\overline{\boldsymbol{P}}=\langle\Psi|\hat{\boldsymbol{P}}| \Psi\rangle=\left(\bar{P}_{x}, \bar{P}_{y}, \bar{P}_{z}\right)=\mathbf{0}
$$

For stationary states, Löwdin's criterion Eq. (1) is satisfied.

Unfortunately, it is difficult to eliminate the electron-electron interaction in systems having more than three electrons. For $N$-electron systems, we need $M=\frac{N(N-1)}{2}$ valuables to describe the electron-electron interaction potentials. The local phase factor $S$ with the $M$ valuables will generate their coupling terms in the calculation of potentials. For example, if we consider a function such as $S=f_{a}\left(\boldsymbol{r}_{1}, \boldsymbol{r}_{2}\right)+f_{b}\left(\boldsymbol{r}_{2}, \boldsymbol{r}_{3}\right)+f_{c}\left(\boldsymbol{r}_{3}, \boldsymbol{r}_{1}\right)$ for three-electron systems to eliminate $\hat{V}_{+}=\frac{1}{\left|\boldsymbol{r}_{1}-\boldsymbol{r}_{2}\right|}+\frac{1}{\left|\boldsymbol{r}_{2}-\boldsymbol{r}_{3}\right|}+\frac{1}{\left|\boldsymbol{r}_{3}-\boldsymbol{r}_{1}\right|}$, there remain some coupling terms like $\left(\frac{\partial S}{\partial x_{1}}\right)^{2}=\left[\frac{\partial f_{a}\left(\boldsymbol{r}_{1}, \boldsymbol{r}_{2}\right)}{\partial x_{1}}\right]^{2}+\left[\frac{\partial f_{c}\left(\boldsymbol{r}_{3}, \boldsymbol{r}_{1}\right)}{\partial x_{1}}\right]^{2}+2\left[\frac{\partial f_{c}\left(\boldsymbol{r}_{1}, \boldsymbol{r}_{2}\right)}{\partial x_{1}} \cdot \frac{\partial f_{a}\left(\boldsymbol{r}_{3}, \boldsymbol{r}_{1}\right)}{\partial x_{1}}\right]$ in Eq. (24). The last term is a type of three-electron operator which cannot be reduced to some simple one-electron operator. Only for two-electron systems, any coupling does not occur. 


\section{SUMMARY}

By adding local phases to the complex wave functions, the repulsive electron-electron interaction has been eliminated from the expectation values of the Hamiltonian in twoelectron systems. Similar to a gauge field transformation, this elimination leads to a oneelectron Hamiltonian operator. The expectation value of the total momentum operator vanishes and Löwdin's criterion Eq. (1) for stationary states is satisfied.

[1] H. Umezawa and G. Vitiello, Quantum Mechanics (Bibliopolis, Napoli, 1985).

[2] J. A. Pople, Int. J. Quantum Chem. 5, 175 (1971).

[3] K. D. Jordan and R. Silbery, Chem. Phys. Lett. 18, 27 (1973).

[4] P. -O. Löwdin, Adv. Quantum Chem. 3, 323 (1967).

[5] E. Brändas, J. Mol. Spect. 27, 236 (1968).

[6] E. Brändas, Int. J. Quantum Chem. 2, 37 (1968).

[7] D. Bohm, Quantum Theory (Prentice-Hall, New York, 1951).

[8] I. J. R. Aitchison and A. J. G. Hey, Gauge Theories in Particle Physics (2nd Ed.) (Adam Hilger, Bristol, 1989). 